\title{
Four-arm robotic sleeve right upper lobectomy
}

\author{
Marion Durand \\ Ramsay Générale de Santé, Thoracic Unit, Hôpital Privé d'Antony, Antony, France \\ Correspondence to: Marion Durand, MD, PhD. Thoracic Unit, Hôpital Privé d'Antony, 1 rue Velpeau, 92160 Antony, France. Email: durandm@me.com.
}

Submitted Aug 16, 2018. Accepted for publication Oct 04, 2018.

doi: $10.21037 / \mathrm{acs} .2018 .11 .02$

View this article at: http://dx.doi.org/10.21037/acs.2018.11.02

\section{Clinical vignette}

We present the case of a 60 -year-old man diagnosed with a proximal right upper lobe (RUL) squamous cell carcinoma after exploration for hemoptysis. The cancer was staged cT3N0M0 as the invasion of the proximal RUL spur was diagnosed during bronchoscopy. The patient was a former smoker (90 pack/year), had lower limb arteritis with a stent and his performance status was preserved (i.e., level 1). His FEV was $75 \%$. He was scheduled for undergoing a fourarm robotic sleeve RU lobectomy and node harvest with an Xi system (Intuitive Surgical, CA, USA).

\section{Surgical technique}

\section{Preparation}

After selective intubation, the patient was placed in a lateral position with a tissue roll under the chest to avoid the hip. As for any lung resection, the axis of the camera arm was placed on the axis of the scapula line. The 30 -degree camera port was placed in the $8^{\text {th }}$ intercostal space (ICS). The right hand was placed in the $7^{\text {th }}$ ICS in the posterior axillary line. Instruments used in this port are either the permanent cautery spatula, the curved scissors, or the needle holder SutureCut ${ }^{\mathrm{TM}}$. The left hand was placed in the $9^{\text {th }}$ ICS in the retro scapula line. The instrument used was fenestrated bipolar forceps. The third arm was placed through the $7^{\text {th }}$ ICS, $5 \mathrm{~cm}$ above the vertebral bone in relation to the scapula end. The instrument used in this port was a ProGrasp ${ }^{\mathrm{TM}}$ forceps. A $15 \mathrm{~mm}$ port access was placed in the $9^{\text {th }}$ ICS, close to the diaphragm insertion. A capnothorax was gently started at $5 \mathrm{mmHg}$ pressure and $10 \mathrm{~L} / \mathrm{min}$ flow.

Two half gauze rolls were inserted, one for each grasper to ensure a non-direct lung retraction. The steps of a routine lobectomy were performed as below.

Firstly, after exploring the cavity, inferior exposure was achieved to perform the triangular ligament dissection, visualization of the inferior pulmonary vein and zone 8 and 9 node harvest. Secondly, zone 7 node harvest and opening of the posterior interbronchial zone was done through posterior exposure. Thirdly, fissure exposure lead to spotting the artery and opening the posterior part of the major fissure, as for ligation of the dorsal artery and hilar node harvest (zones 11, 12) Fourthly, anterior exposure was achieved to control and mechanically suture the anterior mediastinal artery and superior sulcus vein and complete zone 10 node harvest. Finally, after controlling and opening the minor fissure, the superior sulcus bronchus dissection was carried out.

The specific part of the sleeve procedure was then performed. The bronchus was opened in pathologic zone with cold scissors. The specimen was placed in a bag to avoid contamination. The proximal and distal section of the bronchus was performed and the specimen was sent for frozen section analysis. The end-to-end anastomosis was done with two continuous sutures of V-Lock 180 3/0. The first suture was for the posterior wall, starting at 3 o'clock from the outside of the superior border, to 9 o'clock. The anterior wall was subsequently performed from 3 o'clock outside of the lower border, to 9 o'clock.

During the anterior wall suturing, an opening of one of the borders was performed to avoid mismatch of the two borders. The airtight patency of the suture was checked under water. The completion of the node harvest was achieved in section $4 \mathrm{R}$. The cavity was rinsed with saline and the chest closed with a 30 French silicone chest tube through the anterior port (right arm). The specimen in the bag was removed through the port access hole, enlarged as needed. 


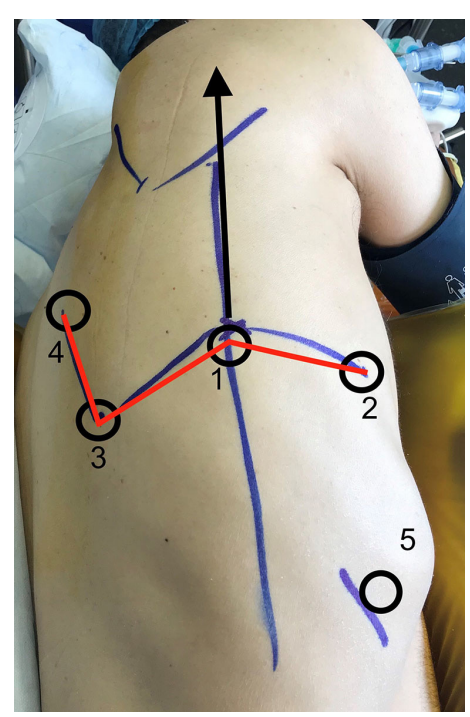

Figure 1 Installation scheme: the numbers show the thoracic ports installation order; the arrow shows the axis of the camera arm; the red line shows the partial "W" shape.

\section{Comments}

The postoperative course was uneventful. The chest tube was removed on postoperative day 2 . The use of the robotic system offers technical conditions comparable to open surgery, mainly due to both visual conditions and three hand wrist tools. The use of the system for complex cases is of great benefit over open surgery for the patient, allowing for the same carcinologic standards but with the benefit of a minimally invasive approach. This technique is different than those recently described by Egberts et al. or Jo et al. $(1,2)$, which were inspired by Veronesi et al. (3) and Cerfolio et al. technique (4). In our technique, two main differences can be highlighted.

Firstly, the positioning of the robotic trocars with a port access more distant/lower allows for a larger range of movement for the assistant and a third hand higher to avoid any internal conflict (Figure 1). Moreover, this positioning is the same for any procedure and is therefore easily reproducible. The second point is about the air patency of the chest and the use of insufflation. We use a low pressure $5 \mathrm{mmHg}$ with $10 \mathrm{~L} / \mathrm{min}$ flow, which is quite low, but enough to enhance exposure. As we use a $15 \mathrm{~mm}$ port rather than $3 \mathrm{~cm}$ incision, airtight patency is ensured. We therefore have the benefit of the capnothorax. The use of V-Lock stitches allows safe tension application, as we do not observe haptic feedback with the system. Two half continuous sutures appear to be a good option to control the posterior wall airtight patency first and secondly to avoid size mismatch on the anterior wall suture at the end.

\section{Acknowledgements}

The author would like to thank Gregory Lemoigne (GLM Prod) for the video editing.

\section{Footnote}

Conflict of interest: The author is proctoring for Intuitive Surgical.

\section{References}

1. Egberts JH, Moller T, Becker T. Robotic-Assisted Sleeve Lobectomy Using the Four-Arm Technique in the DaVinci $\mathrm{Si}{ }^{\circledR}$ and $\mathrm{Xi}{ }^{\circledR}$ Systems. Thorac Cardiovasc Surg 2018. [Epub ahead of print].

2. Jo MS, Kim DY, Jeong JY, et al. Robotic sleeve lobectomy with four arms for lung cancer centrally located in the right lower lobe: a case report. J Cardiothorac Surg 2017;12:108.

3. Veronesi G, Galetta D, Maisonneuve P, et al. Four-arm robotic lobectomy for the treatment of early-stage lung cancer. J Thorac Cardiovasc Surg 2010;140:19-25.

4. Cerfolio RJ, Bryant AS, Skylizard L, et al. Initial consecutive experience of completely portal robotic pulmonary resection with 4 arms. J Thorac Cardiovasc Surg 2011;142:740-6.
Cite this article as: Durand M. Four-arm robotic sleeve right upper lobectomy. Ann Cardiothorac Surg 2019;8(2):286-287. doi: 10.21037/acs.2018.11.02 